\title{
Análisis de la mortalidad por tuberculosis en Medellín, 2012
}

\author{
Liliana Villa', Iván Mauricio Trompa', Fernando Nicolás Montes², \\ Joaquín Guillermo Gómez¹, Carlos Andrés Restrepo ${ }^{1}$ \\ 1 Facultad de Medicina, Universidad de Antioquia, Medellín, Colombia \\ 2 Programa de Control de Tuberculosis y Lepra, Secretaría de Salud de Medellín, Medellín, Colombia
}

Introducción. La tuberculosis es uno de los principales problemas globales de salud pública. Aunque los reportes de la Organización Mundial de la Salud muestran un descenso en las tasas de mortalidad global, Colombia y la ciudad de Medellín no muestran reducciones significativas.

Objetivo. Describir las características sociodemográficas, clínicas y de diagnóstico y tratamiento, de los pacientes fallecidos por tuberculosis en Medellín en el 2012.

Materiales y métodos. Es un estudio descriptivo de las muertes por tuberculosis reportadas en Medellín durante el 2012.

Resultados. El número de muertes fue de 93, de las cuales, 32 (34,4\%) se confirmaron como muertes directas por tuberculosis y $23(24,7 \%)$ como asociadas a esta enfermedad. En $34(61,7 \%)$ de los pacientes hubo alguna enfermedad concomitante, siendo el sida la más importante (18, 32,7 \%). Se detectaron factores de riesgo social como ser habitante de la calle, farmacodependencia o carencia de domicilio fijo, en 32 casos $(58,1 \%)$, y aspectos que afectaron el proceso de atención de los servicios de salud, en 26 (47,2\%). Hubo un retraso de 40 días (rango intercuartílico: 19 a 84) entre el inicio de los síntomas y el diagnóstico. No se encontró retraso significativo en el inicio del tratamiento antituberculoso después del diagnóstico microbiológico; sin embargo, el porcentaje de incumplimiento del tratamiento fue de $64 \%$.

Conclusión. La mortalidad por tuberculosis en Medellín es un problema relevante que está relacionado con retrasos en el diagnóstico de los casos y con el cumplimiento del tratamiento.

Palabras clave: tuberculosis, $\mathrm{VIH}$, mortalidad, factores de riesgo, salud pública, cumplimiento de la medicación.

http://dx.doi.org/10.7705/biomedica.v34i3.2336

\section{Analysis of mortality caused by tuberculosis in Medellín, Colombia, 2012}

Introduction: Tuberculosis is a relevant global public health problem. Although reports of the World Health Organization show decrease in overall mortality rates, Colombia and Medellin show no significant decline.

Objective: To describe the sociodemographic, clinical, diagnosis, and treatment characteristics of patients who died due to tuberculosis in Medellin, Colombia, during 2012.

Materials and methods: A descriptive study of tuberculosis deaths reported in the city.

Results: 93 deaths were identified, of which 32 were confirmed as directly caused by tuberculosis (34.4\%); in 23 deaths (24.7\%) tuberculosis was an associated cause. Co-morbidities were found in 34 patients $(61.7 \%)$, HIV being the most common with 18 cases important( $32.7 \%)$. Social risk factors such as being homeless, drug addiction or having no fixed address were found in 32 cases (58.1\%); and there were deficiencies in the healthcare system in 26 cases (47.2\%). No meaningful delay in the onset of anti-tuberculosis treatment was found after the microbiological diagnosis; however, $64 \%$ of patients did not adhere to treatment.

Conclusion: Mortality caused by tuberculosis in Medellin is a relevant problem associated with delays in diagnosis of the disease and lack of adherence to treatment.

Key words: Tuberculosis, HIV, mortality, risk factors, public health, medication adherence.

http://dx.doi.org/10.7705/biomedica.v34i3.2336

\section{Contribución de los autores:}

Liliana Villa, Iván Trompa y Carlos Restrepo: recolección, procesamiento y análisis de los resultados

Joaquín Gómez y Fernando Montes: análisis de los resultados 
Los Objetivos de Desarrollo del Milenio establecieron metas específicas y medibles de salud pública para el año 2015. En el objetivo relacionado a combatir el $\mathrm{VIH} /$ sida, el paludismo y otras enfermedades, se planteó disminuir en un $50 \%$ la prevalencia y la mortalidad por tuberculosis (1). Aunque los informes de la Organización Mundial de la Salud (OMS) muestran un descenso de $42 \%$ en las tasas de mortalidad desde 1990 (2), las cifras reportadas para Colombia (3) muestran un declive de solo $15 \%$, en tanto que en Medellín no se registran descensos significativos $(4,5)$.

Según el reporte mundial de control de la tuberculosis de la OMS, en el 2010 hubo 8,8 millones de casos de tuberculosis, 1,1 millones de muertes entre pacientes negativos para el VIH y $350 \mathrm{mil}$ muertes por tuberculosis asociadas al VIH. En ese mismo año, el porcentaje de curación a nivel mundial fue de $86 \%$ y, la mortalidad, de $11,7 \%$. En la región de las Américas, la mortalidad por tuberculosis fue de 7,6\% (3). En Medellín, la tasa de incidencia de tuberculosis en todas sus formas en el 2011 fue de 78,9 por 100.000 habitantes, con una la mortalidad de 5,1\% (5); sin embargo, no existen estudios específicos que informen sobre el comportamiento de dicha mortalidad en la ciudad.

El objetivo de este trabajo fue describir las características sociodemográficas, clínicas y de diagnóstico y tratamiento, de los pacientes fallecidos por tuberculosis en Medellín en el año 2012.

\section{Materiales y métodos}

\section{Población del estudio}

Se hizo un estudio descriptivo y retrospectivo de los casos de muertes por tuberculosis reportados en Medellín en el 2012 a través del Sistema de Vigilancia Epidemiológica en Salud Pública (Sivigila) del Instituto Nacional de Salud; este es un sistema de notificación obligatoria iniciado en el 2006 que se nutre de los sistemas de información de cada institución prestadora de servicios de salud, de los consultorios médicos y de los laboratorios de todo el país. Se ha reportado que el porcentaje de confiabilidad en el año 2012 fue

\footnotetext{
Correspondencia:

Carlos Andrés Restrepo, Calle 39 № 64A 35, Edificio Aguas Claras, apartamento 702, Barrio Conquistadores, Medellín, Colombia

Teléfonos: (574) 351 4480, (301) 4251665

restrepocastro@gmail.com

Recibido: 12/03/14; aceptado: 07/05/14
}

de $87,6 \%(6)$, sin mucha variación con respecto a años anteriores (7). Además, se consultaron todos los certificados de defunción de las muertes de personas residentes en la ciudad, a los cuales se accedió por solicitud directa a la Secretaría de Salud de Medellín. Se incluyeron todos los registros de diagnóstico con reporte de muerte por tuberculosis en los certificados de defunción expedidos entre el $1^{\circ}$ de enero y el 31 de diciembre de 2012 (códigos A15 hasta A19 y B200 según la Clasificación Internacional de Enfermedades, CIE-10).

\section{Recolección de la información}

Se llevó a cabo un muestreo por conveniencia y se solicitó copia de las historias clínicas a cada una de las instituciones que participaron en el proceso de atención de los pacientes fallecidos. Se hizo el análisis individual de los casos, para lo cual se creó un comité de análisis conformado por un equipo técnico de expertos en vigilancia epidemiológica, profesionales de medicina interna, infectología, epidemiología y salud pública, integrantes del comité de vigilancia epidemiológica de tuberculosis de Medellín.

En sesiones periódicas, los miembros del comité de análisis hicieron el resumen de cada caso y discutieron todos los aspectos relacionados con el manejo y la evolución de cada paciente hasta llegar a un consenso sobre la causa de la muerte. Siempre se contó con el expediente clínico completo para ser consultado. Se elaboró una base de datos en el programa Access, versión 2010, para la recolección de la información y se verificó periódicamente la completitud y la calidad de la información recolectada.

Definiciones operativas. La tuberculosis se definió como causa básica de la muerte cuando, en la cadena de eventos, la enfermedad tenía una relación directa con la muerte del paciente, y se la consideró causa asociada cuando, a pesar de tener confirmación microbiológica de la enfermedad, el paciente presentaba un evento de salud diferente del que determinó la muerte.

Los miembros del comité consignaban que había habido problemas en la calidad de la atención cuando no se había seguido el protocolo nacional de atención de la tuberculosis (8). En las situaciones en las que el protocolo no era explícito, se recurrió a la última evidencia científica disponible para establecer la calidad de la atención recibida según otras guías internacionales $(9,10)$. 
Los factores relacionados con los servicios de salud que afectaron la calidad de la atención, se agruparon en las siguientes categorías:

1) valoración inadecuada del cuadro clínico por parte del personal médico porque no se hizo búsqueda activa de tuberculosis en pacientes sintomáticos respiratorios o con antecedentes epidemiológicos y cuando no se realizaron estudios adicionales para tuberculosis en pacientes con baciloscopias negativas, pero con signos clínicos o radiológicos evidentes (cavitación, patrón miliar o neumonía apical);

2) exámenes de laboratorio que no fueron solicitados o no estaban disponibles, o recursos insuficientes, por lo que no se solicitaron baciloscopias, cultivos, radiografías o métodos diagnósticos adicionales, según indica la guía nacional;

3) falta de oportunidad en la atención médica en los casos en que el paciente requirió más de dos consultas antes del diagnóstico o cuando faltó la revisión de los resultados de cultivo solicitados;

4) intervenciones o tratamientos inadecuados por inicio tardío del tratamiento, dosificación inadecuada del tratamiento antituberculoso, interacciones farmacológicas previsibles o manejo inadecuado de la toxicidad de los medicamentos;

5) interrogatorio o examen físico deficientes por no haberse registrado en la historia clínica los signos o síntomas del paciente;

6) barreras administrativas para la atención, pues se presentaron dificultades para la práctica de las pruebas diagnósticas o terapéuticas, a pesar de la prescripción médica oportuna, y

7) dificultades en el traslado o en el trabajo en equipo del personal de salud.

Se consideró incumplimiento del tratamiento cualquier interrupción documentada de la toma de los medicamentos, independientemente de la causa. La resistencia a los fármacos se definió como la disminución de la sensibilidad a uno o más fármacos de primera línea. La tuberculosis multirresistente se definió como la presencia de resistencia tanto a la rifampicina como a la isoniacida (11).

Se consideró como factor de riesgo social cualquier situación de aislamiento social de un paciente, como el alcoholismo (sin considerar la cantidad de alcohol tomada diariamente), el consumo activo de drogas psicoactivas, la estancia en prisión y la ausencia de domicilio fijo. Se consideró inadaptación social "toda anomalía del comportamiento social por falta de apoyos familiares, económicos, materiales, y que conlleva abandono físico, baja autoestima y baja afectividad" (12).

\section{Análisis}

Se hizo un análisis descriptivo de la información sociodemográfica y clínica, así como de los casos de muerte en pacientes con tuberculosis, incluida la infección concomitante con VIH, y se clasificaron las muertes como causadas directamente por tuberculosis o asociadas a ella. Todas las historias clínicas se revisaron en el comité para determinar la calidad de la atención. Los datos se procesaron en el paquete estadístico SPSSTM, versión 20, utilizando medidas de distribución de frecuencia para las variables cualitativas y las medidas de tendencia central, dispersión y posición para las variables cuantitativas.

\section{Resultados}

Se encontraron 164 muertes por tuberculosis a partir de la información consolidada obtenida de los certificados de defunción y el Sivigila; solo en 93 de estos casos se obtuvieron las historias clínicas para el análisis y en 44 de estos, se obtuvo la información sociodemográfica básica (cuadro 1). Se confirmaron como muertes directas por tuberculosis, $32(34,4 \%)$ de los casos analizados y en $23(24,7 \%)$ de ellos se la consideró como causa asociada. Se descartó la tuberculosis como causa directa de la muerte o como causa asociada a esta en 38 (40,9\%) casos, lo que evidencia la concordancia entre los registros estadísticos citados y lo encontrado en el estudio en 55 (59,1\%) de los casos (figura 1).

Entre los 55 casos clasificados como muerte por causa directa 0 asociada a la tuberculosis, 36 $(65,5 \%)$ correspondían a hombres y $19(34,5 \%)$ a mujeres; la edad media fue de $43,5 \pm 17,1$ años,

Cuadro 1. Características sociodemográficas de los casos no analizados

\begin{tabular}{lc}
\hline Variable & $\begin{array}{c}\text { Número (\%) } \\
\mathrm{N}=44\end{array}$ \\
\hline Edad en años & $54,05 \pm 19,9$ \\
Hombres & $26(56)$ \\
Mujeres & $18(44)$ \\
Afiliación al SGSSS & \\
Subsidiado & $23(52,2)$ \\
Contributivo & $14(31,8)$ \\
No afiliado & $7(16)$ \\
\hline
\end{tabular}

SGSSS: Sistema General de Seguridad Social en Salud 




Figura 1. Diagrama de selección de casos

siendo el 70,9\% de los pacientes menores de 50 años (cuadro 2). En los pacientes en quienes la tuberculosis se confirmó como enfermedad asociada, las principales causas directas de muerte fueron otras infecciones oportunistas asociadas al $\mathrm{VIH}$ en $6(32,7 \%)$ casos y, a enfermedades cardiovasculares, en 4 (12,7\%) (cuadro 3).

De las muertes directas o asociadas a la tuberculosis, 30 (55\%) casos fueron diagnosticados en el hospital y, de ellos, 23 (76,6 \%) presentaban enfermedades concomitantes de base y $12(40 \%)$ presentaban algún factor de riesgo social, como ser habitante de calle, ser farmacodependiente o no tener domicilio fijo (cuadro 2). A los 25 (45\%) pacientes restantes se les hizo el diagnóstico ambulatoriamente y, de ellos, 9 (36 \%) tenían enfermedades concomitantes de base, siendo la infección por VIH la más frecuente en 8 (88\%). En 21 (84\%) pacientes se encontró algún factor de riesgo social (cuadro 2). En 16 (64\%) de los casos con diagnóstico ambulatorio, se encontró el antecedente de incumplimiento del tratamiento.

Se encontraron aspectos relacionados con los servicios de salud que afectaron el proceso de atención en $26(47,2 \%)$ pacientes, y los más frecuentes fueron la inadecuada valoración del cuadro clínico en 15 (27,3 \%) y la no realización de los exámenes de laboratorio necesarios en 14 $(25,5 \%)$ (cuadro 4). Hubo un retraso general de 40 días (rango intercuartílico: 19 a 84) entre el inicio de los síntomas y el diagnóstico.
Cuadro 2. Características sociodemográficas y clínicas de los casos de muertes relacionadas con tuberculosis

\begin{tabular}{|c|c|}
\hline Variable & $\begin{array}{c}\text { Número (\%) } \\
\mathrm{N}=55\end{array}$ \\
\hline \multicolumn{2}{|l|}{ Afiliación al SGSSS } \\
\hline Subsidiado & $25(45,4)$ \\
\hline Contributivo & $18(32,7)$ \\
\hline No afiliado & $12(21,8)$ \\
\hline Factor de riesgo social & $32(58,1)$ \\
\hline Farmacodependencia & $22(40)$ \\
\hline Habitante de calle & $12(21,8)$ \\
\hline Ausencia de domicilio fijo & $1(1,8)$ \\
\hline Alcoholismo & $1(1,8)$ \\
\hline Privado de libertad & $1(1,8)$ \\
\hline Historia previa de tuberculosis & $26(47)$ \\
\hline \multicolumn{2}{|l|}{ Clasificación de tuberculosis } \\
\hline Pulmonar & $38(69,1)$ \\
\hline Extrapulmonar & $17(30,9)$ \\
\hline \multicolumn{2}{|l|}{ Hallazgos radiológicos* } \\
\hline Anormal $^{* *}$ & $45(81,8)$ \\
\hline Cavitación & $21(38,2)$ \\
\hline Normal & $6(10,9)$ \\
\hline Enfermedades concomitantes & $34(61,8)$ \\
\hline VIH/sida & $18(32,7)$ \\
\hline Enfermedad cardiovascular & $7(12,7)$ \\
\hline Diabetes & $4(7,3)$ \\
\hline Enfermedad renal & $4(7,3)$ \\
\hline Cáncer & $4(7,3)$ \\
\hline Enfermedad pulmonar obstructiva crónica & $5 \quad(9,1)$ \\
\hline Enfermedad hepática & $4(7,3)$ \\
\hline \multicolumn{2}{|l|}{ Síntomas } \\
\hline Tos & $42(76,4)$ \\
\hline Disnea & $36(65,5)$ \\
\hline Astenia & $36(65,5)$ \\
\hline Fiebre & $34(61,8)$ \\
\hline Expectoración & $34(61,8)$ \\
\hline Adinamia & $34(61,8)$ \\
\hline Pérdida de peso & $33(60)$ \\
\hline Hemoptisis & $4(7,3)$ \\
\hline Otros & $13(23,6)$ \\
\hline
\end{tabular}

SGSSS: Sistema General de Seguridad Social en Salud

* Datos extraídos del total de radiografías disponibles

** Presencia de infiltración alveolar, adenopatías hiliares o consolidación

Cuadro 3. Causa de muerte en los casos de tuberculosis confirmada como causa asociada a la muerte

\begin{tabular}{lc}
\hline Causa de muerte & $\mathbf{n}$ \\
\hline VIH/sida & 6 \\
Enfermedad cardiovascular & 4 \\
Neumonía* & 3 \\
Cáncer & 2 \\
Sepsis & 2 \\
Diabetes mellitus & 1 \\
Enfermedad pulmonar obstructiva crónica & 1 \\
Insuficiencia renal crónica & 1 \\
No especificada & 1 \\
Agresión con arma de fuego & 1 \\
Total & $\mathbf{2 2}$ \\
\hline
\end{tabular}

* En muestras de lavado broncoalveolar se confirmó la acción de otros microorganismos como causa básica.

** En muestras de hemocultivos se confirmó la acción de otros microorganismos como causa básica. 
Cuadro 4. Distribución de los factores relacionados con los servicios de salud que afectaron el proceso de atención en los casos de muertes relacionadas con la tuberculosis

\begin{tabular}{lrr}
\hline $\begin{array}{l}\text { Factores relacionados con la calidad } \\
\text { de la atención }\end{array}$ & $\mathbf{n}$ & $\%$ \\
\hline Inadecuada valoración del cuadro clínico & 15 & 27,3 \\
Falta de exámenes de laboratorio & 14 & 25,5 \\
Recursos insuficientes o inadecuados & 13 & 23,6 \\
para el nivel de atención & & \\
Falta de oportunidad en la atención & 13 & 23,6 \\
Intervenciones o conductas inadecuadas & 13 & 23,6 \\
Tratamiento inadecuado & 12 & 21,8 \\
Deficiencias en la entrevista & 11 & 20,0 \\
Nivel de atención inadecuado & 10 & 18,2 \\
Deficiencias del examen físico & 8 & 14,5 \\
Barreras administrativas & 4 & 7,3 \\
Dificultades en el traslado & 1 & 1,8 \\
Dificultades en el trabajo en equipo & 1 & 1,8 \\
\hline
\end{tabular}

El diagnóstico de tuberculosis se hizo por baciloscopia en 39 (70,1\%) casos, por cultivo en 11 (20\%), hubo biopsia en tres $(5,4 \%)$ y se sospechó por signos clínicos en $2(3,6 \%)$. En $3(5,3 \%)$ de los pacientes se practicaron estudios de biología molecular como pruebas complementarias.

En 18 (32,7\%) pacientes se demostró la presencia concomitante de infección por $\mathrm{VIH}$; la forma extrapulmonar de la tuberculosis fue la presentación clínica más frecuente de la enfermedad en 11 $(61,1 \%)$ casos.

De los 7 (38,9 \%) pacientes con infección concomitante por VIH y tuberculosis pulmonar confirmada, se reportaron radiografías de tórax normales en $4(57,1 \%$ ) y bacilo de Koch en esputo negativo en 3 (42,8\%). De estos tres casos, en dos se hizo el diagnóstico con base en el cultivo y por estudios de biología molecular en lavado broncoalveolar en el otro.

La mediana de tiempo entre el inicio de los síntomas y el diagnóstico en los pacientes con infección concomitante con VIH fue de 52 días (rango intercuartílico: 25 a 289) y de 96 días (rango intercuartílico: 40 a 152) para los pacientes con formas extrapulmonares, radiografías reportadas como normales y baciloscopia negativa en esputo.

Se encontró algún tipo de resistencia a los fármacos antituberculosos en $6(10,9 \%)$ casos y solo hubo uno de tuberculosis multirresistente. Cuatro $(7,3 \%)$ del total de pacientes no recibieron el tratamiento oportunamente, es decir, hubo una diferencia mayor de dos días entre el diagnóstico y el inicio del tratamiento, con una mediana de tiempo de 11 días (rango intercuartílico: 6,5 a 26).

\section{Discusión}

La tuberculosis en Medellín sigue siendo una enfermedad de alta prevalencia, con tasas de mortalidad que han presentado poca disminución en los últimos años. En este estudio hallamos algunos factores comunes a los pacientes fallecidos por esta enfermedad.

En primer lugar, la mayoría de los diagnósticos se hizo en el hospital (55\%). En este grupo de pacientes predominó la presencia de enfermedades crónicas concomitantes, comparado con el grupo de pacientes diagnosticados en los servicios ambulatorios (76 Vs. $36 \%$, respectivamente). Estas enfermedades concomitantes se han relacionado con el aumento de la mortalidad atribuible a la tuberculosis en otros estudios $(13,14)$. Asimismo, el diagnóstico hospitalario se ha asociado a una presentación clínica en estadios más avanzados de la tuberculosis (15), lo que probablemente refleja una baja captación de sintomáticos respiratorios en el ámbito ambulatorio y la falta de reconocimiento de los signos de gravedad por parte de los pacientes y del grupo médico (16-19).

En el grupo de pacientes diagnosticados en el servicio ambulatorio, hubo menos incidencia de enfermedades concomitantes debido, quizá, a la captación de pacientes en estadios más tempranos de la enfermedad. La mortalidad en este grupo podría explicarse por la falta de cumplimiento del tratamiento debido a la alta proporción de factores de riesgo social ( $88 \%$ ), lo que lleva a la progresión de cualquier enfermedad no tratada y a una mayor mortalidad, como se ha reportado en otros estudios $(12,20)$.

En segundo lugar, se evidenciaron falencias en la calidad de la atención prestada por los servicios de salud en el $47,2 \%$ de los casos, siendo las más frecuentes aquellas relativas al enfoque clínico y el diagnóstico de los pacientes (25\%). En estudios similares, como una serie reportada en Rusia, se ha determinado que solo el $18 \%$ de los casos letales se diagnostica adecuadamente y que las principales dificultades se deben al deficiente reconocimiento de los factores de riesgo y los signos de alarma, así como a la interpretación de los estudios diagnósticos $(21,22)$, lo que hace necesario tomar medidas para mejorar la calidad de la atención en salud y también la educación, desde la formación universitaria hasta la capacitación continuada del personal de salud, así como crear centros de referencia para la atención de pacientes con tuberculosis, entre otras. 
En más del $20 \%$ de los casos evaluados, el diagnóstico se hizo solo por medio de cultivos. Esto implica una demora en el inicio del tratamiento que pudo haber afectado el resultado clínico. En ese sentido, la utilización de pruebas rápidas como las de biología molecular (ya disponibles en nuestro medio), puede agilizar el diagnóstico y, por ende, disminuir la mortalidad $(23,24)$. En nuestra serie, el método molecular fue el único estudio que permitió el diagnóstico en 5,3\% de los casos; sin embargo, a la gran mayoría de pacientes no se les hizo este tipo de pruebas, muy probablemente por falta de conocimiento sobre su disponibilidad y por su costo excesivo en el país. Por lo tanto, las instituciones de salud correspondientes deberían promocionar la disponibilidad de dichas pruebas y regular su costo.

El $32 \%$ de los pacientes fallecidos evaluados en este análisis, tenían tanto tuberculosis como infección por $\mathrm{VIH}$, datos que coinciden con las estadísticas mundiales que muestran cómo la tuberculosis es la causa de muerte de una de cada tres personas con sida (3). Hubo un retraso evidente en la mediana del tiempo de diagnóstico de la tuberculosis en los pacientes sin VIH comparados con los positivos para VIH (40 Vs. 52 días); esta diferencia fue mayor en aquellos con formas extrapulmonares (96 días). En un metaanálisis en que se evaluó el retraso en el diagnóstico de tuberculosis pulmonar, se concluyó que el tiempo promedio entre el inicio de los síntomas y el diagnóstico fue de 28,5 días en los países de bajos ingresos y de 21,5 días en los países de ingresos altos (25). Nuestros resultados denotan la ausencia de sospecha en torno a los signos clínicos de la tuberculosis en pacientes que frecuentemente presentan manifestaciones atípicas y pruebas microbiológicas iniciales negativas (26-28). En este sentido, es pertinente la creación de un programa nacional encargado del manejo integral del paciente con infección concomitante de VIH y tuberculosis.

En tercer lugar, no existió un retraso significativo en el inicio del tratamiento una vez hecho el diagnóstico de tuberculosis. Sin embargo, en nuestra serie sí hubo un alto porcentaje de incumplimiento de dicho tratamiento (64\%), la mayoría entre pacientes con factores de riesgo social. Esto podría evidenciar fallas del programa de control de la tuberculosis para el reconocimiento de pacientes con alto riesgo de abandono, lo que influye en el resultado, como se ha demostrado previamente (29). Esto plantea la necesidad de revisar el programa de tuberculosis en la ciudad para fortalecer e implementar estrategias adicionales, como subsidios de alimentación y transporte, asignación de pares e institucionalización en los casos que se requieran (30-33).

Otro hallazgo relevante fue la imprecisión de los registros estadísticos nacionales para la definición de casos de mortalidad por tuberculosis, pues la concordancia entre lo reportado y lo observado fue de $60 \%$ únicamente. Esto también se ha reportado en otros países, probablemente debido a que dichas estadísticas se basan solo en la revisión de las bases de datos demográficas, de los programas de tuberculosis o los registros nacionales de defunciones, cuya precisión no se verifica y que no tienen criterios estandarizados sobre la calidad del diagnóstico principal o las causas definitivas de mortalidad $(34,35)$. Lo anterior hace necesario que se fortalezcan los sistemas de información de la vigilancia epidemiológica en el país.

Se encontró algún tipo de resistencia a los fármacos antituberculosos en el 10,9 \% de los casos, sin embargo, solo hubo un caso de tuberculosis multirresistente. Estos hallazgos sugieren que, a pesar de que la tuberculosis multirresistente en Colombia mostró un incremento de 55,8 \% entre el año 2001 (43 casos) y el 2009 (120 casos) (11), aún no representa una causa importante de mortalidad en nuestra población, como sí se ha mostrado en otros estudios (36).

Hasta donde sabemos, es la primera vez que se aplica un análisis de este tipo para la mortalidad por tuberculosis en Colombia. Esta metodología es novedosa, pues se estableció la veracidad del diagnóstico de la tuberculosis caso por caso, siguiendo criterios microbiológicos y clínicos estrictos. Este modelo demostró ser útil porque aportó elementos clave para la valoración de la calidad de la atención de los pacientes a partir de la evaluación exhaustiva de la historia clínica. Con base en esta experiencia valdría la pena implementar este tipo de análisis para supervisar la calidad del programa y garantizar su mejoramiento.

Entre las debilidades del estudio se debe señalar que no se logró hacer el análisis de los retrasos atribuibles a los pacientes y los originados en el acceso a los servicios de salud, debido a la deficiencia de los registros clínicos, ya que se necesitaban fuentes adicionales de información como el registro de las visitas domiciliarias y las entrevistas a los familiares. Tampoco se analizaron todos los casos de mortalidad debido a la falta de registros clínicos completos, lo que impidió generar 
datos epidemiológicos precisos sobre la tasa de mortalidad por tuberculosis. Por último, dada la naturaleza retrospectiva del estudio, los datos pueden estar sujetos a sesgos, pues provienen de registros médicos ya existentes.

En conclusión, la mortalidad por tuberculosis en Medellín es un problema relevante. En los casos evaluados, se apreciaron factores como el retraso en el diagnóstico, la inadecuada valoración del cuadro clínico, una gran cantidad de diagnósticos hospitalarios, el uso insuficiente de métodos diagnósticos rápidos, la infección de tuberculosis y $\mathrm{VIH}$, y la presencia de factores de riesgo social en los pacientes. Además, el sistema de salud tiene falencias en la implementación de estrategias para lograr cumplimiento del tratamiento. Los resultados de este estudio constituyen un insumo para la toma de decisiones que apunten al mejoramiento del programa de control de la tuberculosis.

\section{Agradecimientos}

A la Secretaría de Salud de Medellín y al Centro NACER de la Universidad de Antioquia, por permitir el desarrollo de este estudio a partir de la información recolectada en el marco del proyecto "Así te ves salud" en su componente de vigilancia epidemiológica de la mortalidad por tuberculosis.

\section{Conflicto de intereses}

No hay conflictos de intereses para señalar por parte de los autores.

\section{Financiación}

Este proyecto fue financiado por la Secretaría de Salud de Medellín a través del contrato interadministrativo 4600042998 del 2012.

\section{Referencias}

1. World Health Organization. Plan mundial para detener la tuberculosis 2006-2015. Fecha de consulta: 24 de octubre de 2013. Disponible en: http://www.stoptb.org/assets/ documents/global/plan/GPII_SPversionfinale.pdf.

2. World Health Organization. Global tuberculosis report 2012. Fecha de consulta: 22 de octubre de 2013. Disponible en: http://www.who.int/tb/publications/global_report/en/.

3. World Health Organization. Global tuberculosis control 2011. Fecha de consulta: 22 de octubre de 2013. Disponible en: http://www.who.int/tb/publications/global_report/2011/en/.

4. Segura AM, Rey JJ, Arbelaéz MP. Trends of tuberculosis related mortality and hospital discharges before and after the implementation of the health sector reform, Colombia, 1985-1999. Biomédica. 2004;24(Supl.1):115-23.

5. Dirección Seccional de Salud de Antioquia. Boletín Información para la Acción, marzo 2012. Fecha de consulta:
22 de octubre de 2013. Disponible en: http://www.dssa.gov. $\mathrm{co} /$ media_ddssa/dssa.gov.co/documentos/bia-final.pdf.

6. López JG. Acta de informe de gestión -informe de empalme 2012. Fecha de consulta: 22 de octubre de 2013. Disponible en:http://www.ins.gov.co/control-ytransparencia/SiteAssets/ Paginas/rendici\%C3\%B3n-de-cuentas/INFORME\%20 EMPALME\%202012.pdf.

7. López DM. Un sistema de vigilancia en salud pública para alertas tempranas - 2008. Fecha de consulta: 25 de enero de 2014. Disponible en: http://www.icesi.edu.co/revistas/ index.php/sistemas_telematica/article/view/983.

8. Ministerio de la Protección Social. Guía de atención de la tuberculosis pulmonar y extrapulmonar -2003. Fecha de consulta: 21 de octubre de 2013. Disponible en: http://www. nacer.udea.edu.co/pdf/libros/guiamps/guias11.pdf.

9. World Health Organization. Guidelines for treatment of tuberculosis, fourth edition - 2006. Fecha de consulta: 25 de enero de 2014. Disponible en: http://www.who.int/tb/ publications/2010/9789241547833/en/

10. Infectious Diseases Society of America. International Standards for Care of Tuberculosis. Fecha de consulta: 25 de enero de 2014. Disponible en: http://www.idsociety. org/IDSA/Site_Map/Guidelines/Patient_Care/IDSA_ Practice_Guidelines/Infections_by_Organism/HIV/AIDS/ International_Standards_for_Care_of_Tuberculosis.aspx.

11. Instituto Nacional de Salud. Guía para el manejo programático del paciente con tuberculosis farmacorresistente en Colombia-2013. Fecha de consulta: 24 de octubre de 2013. Disponible en: http://www.ins.gov.co/lineasde-accion/SubdireccionVigilancia/micobacterias/ LineamientosmanejodeTuberculosisFarmacorresistente.pdf.

12. Anibarro L, Lires JA, Iglesias F, Vilariño C, Baloria A, de Lis JM, et al. Factores sociales de riesgo para la falta de cumplimiento terapéutico en pacientes con tuberculosis en Pontevedra. Gac Sanit. 2004;18:38-44. http://dx.doi.org/10. 1590/S0213-91112004000100007

13. Yen YF, Rodwell TC, Yen MY, Shih HC, Hu BS, Li LH, et al. DOT associated with reduced all-cause mortality among tuberculosis patients in Taipei, Taiwan, 2006-2008. Int J Tuberc Lung Dis. 2012;16:178-84. http://dx.doi.org/10.5588/ ijtld.11.0034

14. Sbrana E, Grise J, Stout C, Aronson J. Co-morbidities associated with tuberculosis in an autopsy case series. Tuberc Edinb Scotl. 2011;91(Suppl.1):S38-42. http://dx.doi. org/10.1016/j.tube.2011.10.008

15. Lin CY, Lin WR, Chen TC, Lu PL, Huang PM, Tsai ZR, et al. Why is in-hospital diagnosis of pulmonary tuberculosis delayed in southern Taiwan? J Formos Med Assoc. 2010; 109:269-77. http://dx.doi.org/10.1016/S0929-6646(1060052-6

16. Lock WA, Ahmad RA, Ruiter RA, van der Werf MJ, Bos AE, Mahendradhata $Y$, et al. Patient delay determinants for patients with suspected tuberculosis in Yogyakarta province, Indonesia. Trop Med Int Health. 2011;16:1501-10. http://dx.doi.org/10.1111/j.1365-3156.2011.02864.x

17. Tattevin P, Che D, Fraisse P, Gatey C, Guichard C, Antoine $\mathbf{D}$, et al. Factors associated with patient and health care system delay in the diagnosis of tuberculosis in France. Int J Tuberc Lung Dis. 2012;16:510-5. http://dx.doi. org/10.5588/ijtld.11.0420 
18. Verhagen LM, Kapinga R, van Rosmalen-Nooijens K. Factors underlying diagnostic delay in tuberculosis patients in a rural area in Tanzania: A qualitative approach. Infection. 2010;38:433-46. http://dx.doi.org/10.1007/s15010-010-0051-y

19. Kiwuwa MS, Charles K, Harriet MK. Patient and health service delay in pulmonary tuberculosis patients attending a referral hospital: A cross-sectional study. BMC Public Health. 2005;5:122. http://dx.doi.org/10.1186/1471-2458-5-122

20. Paul D, Busireddy A, Nagaraja SB, Satyanarayana S, Dewan PK, Nair SA, et al. Factors associated with delays in treatment initiation after tuberculosis diagnosis in two districts of India. PLoS ONE. 2012;7:e39040. http://dx.doi. org/10.1371/journal.pone.0039040

21. Surkova LK, Dius'mikeeva MI, Puchkov AF. Errors in the diagnosis of tuberculosis in general hospitals. Klin Med (Mosk). 1991;69:40-3.

22. Storla DG, Yimer S, Bjune GA. A systematic review of delay in the diagnosis and treatment of tuberculosis. BMC Public Health. 2008;8:15. http://dx.doi.org/10.1186/14712458-8-15

23. Yoon C, Cattamanchi A, Davis JL, Worodria W, den Boon S, Kalema N, et al. Impact of Xpert MTB/RIF testing on tuberculosis management and outcomes in hospitalized patients in Uganda. PLoS One. 2012;7:e48599. http://dx. doi.org/10.1371/journal.pone.0048599

24. Lima AS, Duarte RS, Montenegro LML, Schindler HC. Rapid detection and differentiation of mycobacterial species using a multiplex PCR system. Rev Soc Bras Med Trop. 2013;46:447-52. http://dx.doi.org/10.1590/0037-8682-00972013

25. Sreeramareddy CT, Panduru KV, Menten J, van den Ende J. Time delays in diagnosis of pulmonary tuberculosis: A systematic review of literature. BMC Infect Dis. 2009;9:91. http://dx.doi.org/10.1186/1471-2334-9-91

26. Silva DR, Müller AM, Dalcin PTR. Factors associated with delayed diagnosis of tuberculosis in hospitalized patients in a high TB and HIV burden setting: A cross-sectional study. BMC Infect Dis. 2012;12:57. http://dx.doi.org/10.1186/14712334-12-57

27. Thakur R, Murhekar M. Delay in diagnosis and treatment among TB patients registered under RNTCP Mandi, Himachal Pradesh, India, 2010. Indian J Tuberc. 2013;60:37-45.
28. Balabanova Y, Tchernyshev V, Tsigankov I, Maximova S, Mikheeva N, Fedyukovitch L, et al. Analysis of undiagnosed tuberculosis-related deaths identified at post-mortem among HIV-infected patients in Russia: A descriptive study. BMC Infect Dis. 2011;11:276. http://dx.doi.org/10.1186/14712334-11-276

29. Ahmad RA, Mahendradhata Y, Utarini A, de Vlas SJ. Diagnostic delay amongst tuberculosis patients in Jogjakarta Province, Indonesia, is related to the quality of services in DOTS facilities. Trop Med Int Health. 2011;16:412-23. http:// dx.doi.org/10.1111/j.1365-3156.2010.02713.x

30. Thiam S, LeFevre AM, Hane F, Ndiaye A, Ba F, Fielding $\mathrm{KL}$, et al. Effectiveness of a strategy to improve adherence to tuberculosis treatment in a resource-poor setting: $A$ cluster randomized controlled trial. JAMA. 2007;297:380-6. http://dx.doi.org/110.1001/jama.297.4.380

31. Volmink J, Garner P. Systematic review of randomized controlled trials of strategies to promote adherence to tuberculosis treatment. BMJ. 1997;315:1403-6. http://dx.doi. org/10.1136/bmj.315.7120.1403

32. Volmink J, Garner P. Interventions for promoting adherence to tuberculosis management. Cochrane Database Syst Rev. 2000;4:CD000010. http://dx.doi.org/10.1002/14651858. CD000010

33. Lutge EE, Wiysonge CS, Knight SE, Volmink J. Material incentives and enablers in the management of tuberculosis. Cochrane Database Syst Rev. 2012;1:CD007952. http:// dx.doi.org/10.1002/14651858.CD007952.pub2

34. Rodríguez H, Ciriacos C, Piñeyrúa $M$, Logaldo $R$, González D. Calidad del registro en el certificado de defunción en un hospital público de referencia. Montevideo, Uruguay, octubre-noviembre 2009. Rev Médica Urug. 2010;26:216-23.

35. Pritt BS, Hardin NJ, Richmond JA, Shapiro SL. Death certification errors at an academic institution. Arch Pathol Lab Med. 2005;129:1476-9. http://dx.doi.org/10.1043/15432165(2005)129[1476:DCEAAA]2.0.CO;2

36. Horne DJ, Hubbard R, Narita M, Exarchos A, Park DR, Goss $\mathrm{CH}$. Factors associated with mortality in patients with tuberculosis. BMC Infect Dis. 2010;10:258. http://dx.doi. org/10.1186/1471-2334-10-258 